\title{
Similar Clinical Outcomes for THAs With and Without Prior Periacetabular Osteotomy
}

\author{
Derek F. Amanatullah MD, PhD, Louis Stryker MD, Perry Schoenecker MD, \\ Michael J. Taunton MD, John C. Clohisy MD, Robert T. Trousdale MD, \\ Rafael J. Sierra MD
}

Received: 20 March 2014/Accepted: 16 October 2014/Published online: 31 October 2014

(C) The Association of Bone and Joint Surgeons (B) 2014

\begin{abstract}
Introduction Some patients opt to undergo conversion to a THA for continued pain or progression of hip arthritis after periacetabular osteotomy. Whether patients are at greater risk for postoperative complications, revision THA, poor clinical outcomes, or compromised radiographic results after periacetabular osteotomy is debatable.

Questions/purposes When compared with a matched cohort of patients who underwent THAs for developmental dysplasia of the hip (DDH) without previous periacetabular osteotomy, we asked whether a THA after a periacetabular osteotomy has (1) a higher complication rate, (2) a higher
\end{abstract}

One of the authors certifies that he (DFA) has received amounts less than USD 10,000 from Medscape (New York, NY, USA), less than USD 10,000 from Sanofi (Paris, France), less than USD 10,000 from AO North America (Paoli, PA, USA), less than USD 10,000 from Trimed (Santa Clara, CA, USA), less than USD 10,000 from Acumed (Hillsboro, OR, USA), and less than USD 10,000 from DePuySynthes (Warsaw, IN, USA) during the study period. One of the authors (MJT) certifies that he has received an amount less than USD 10,000 from Don Joy Orthopedics (Vista, CA, USA) during the study period. One of the authors (JCC) certifies that he has received amounts less than USD 10,000 from Biomet (Warsaw, IN, USA) and less than USD 10,000 from Pivot Medical (Sunnyvale, CA, USA), USD 100,000 to USD 1,000,000 from Zimmer (Warsaw, IN, USA) and less than USD 10,000 from Wright Medical Technologies (Memphis, TN, USA) during the study period. One of the authors (RTT) certifies that he has received USD 100,000 to USD 1,000,000 from DePuy-Synthes (Warsaw, IN, USA) during the study period. One of the authors (RJS) certifies that he has received USD 10,000 to USD 100,000 from Biomet (Warsaw, IN, USA) during the study period.

All ICMJE Conflict of Interest Forms for authors and Clinical Orthopaedics and Related Research ${ }^{\mathbb{R}}$ editors and board members are on file with the publication and can be viewed on request.

Each author certifies that his or her institution approved the human protocol for this investigation that all investigations were conducted in conformity with ethical principles of research, and that informed consent for participation in the study was obtained. likelihood of resulting in revision THA, (3) comparable improvements in Harris hip score, and (4) comparable radiographic results.

Patients and Methods A multicenter retrospective review of 562 patients undergoing 645 periacetabular osteotomies was performed. Twenty-three hips in 22 patients underwent a THA after periacetabular osteotomy. The patients were matched for age, sex, and BMI with 23 hips in 23 patients with DDH undergoing THA without a history of periacetabular osteotomy. Minimum followup for both groups of patients was 2 years (mean, $10 \pm 4$ years and $6 \pm 4$ years, respectively). Comparisons were made to answer the study

This study was performed at Mayo Clinic, Rochester, MN, USA, and Washington University, St Louis, MO, USA.

\section{F. Amanatullah \\ Department of Orthopedic Surgery, Stanford University, Redwood City, CA, USA}

M. J. Taunton, R. T. Trousdale, R. J. Sierra ( $\square)$

Department of Orthopedic Surgery, Mayo Clinic, 200 First Street

SW, Gonda 14, Rochester, MN 55905, USA

e-mail: sierra.rafael@mayo.edu

L. Stryker

OrthoCarolina, Charlotte, NC, USA

P. Schoenecker, J. C. Clohisy

Department of Orthopaedic Surgery, Washington University

School of Medicine, St. Louis, MO, USA 
questions based on a retrospective review from prospectively maintained registries of clinical and radiographic information at two participating centers.

Results With the numbers available, there was no difference in complication or revision rates between the two groups $(\mathrm{p}=0.489$ and 1.000 , respectively); however, a post hoc power analysis showed our study was underpowered to detect a difference in the rate of postoperative complications or revision THA. There was marked improvement in Harris hip score with THA after periacetabular osteotomy $(p<0.001)$ and THA for DDH $(p<0.001)$, but there was no difference $(p=0.265)$ in the Harris hip score at final followup between either group. The acetabular component was placed at a mean of $17^{\circ}$ more retroversion during THA after periacetabular osteotomy compared with THA for DDH $(\mathrm{p}=0.002)$.

Conclusions This study did not detect any differences in the clinical outcomes in patients undergoing THA after periacetabular osteotomy done with a modern abductorsparing approach when compared with a matched cohort undergoing THA for DDH. However, even with patients tallied across two high-volume centers during nearly 15 years, our study was underpowered to detect potentially important differences between the THA after periacetabular osteotomy group and the THA for DDH group. The data in this report are suitable as pilot data for future studies and for systematic reviews. Larger multicenter studies are needed to understand how the technical challenges of THA after periacetabular osteotomy affect postoperative complications and revision THA.

Level of Evidence Level III, therapeutic study.

\section{Introduction}

Developmental dysplasia of the hip (DDH) is recognized as a cause of pain and limitation that predisposes patients to early arthritic changes [16]. Symptomatic patients with DDH may benefit from joint preservation. A periacetabular osteotomy improves pain and function in the majority of these patients [7]. It also improves the mechanics of the hip, protects the labrum, provides symptomatic pain relief, and may prevent the development of osteoarthritis. Despite its reported benefits, some patients will undergo THA after periacetabular osteotomy for continued pain and progression of hip arthritis [19].

Previous surgery may compromise the results of hip or knee arthroplasty. High tibial osteotomy, unicompartmental arthroplasty, and knee arthroscopy have been shown to compromise the results of subsequent TKA [1, 3, 9-11, 14, 15, 23]. Furthermore, previous femoral osteotomies and acetabular or femoral fractures make performing a THA more difficult and compromise the results of subsequent
THA $[2,6,12,13,22]$. One study reported the results of 41 patients who underwent THA after periacetabular osteotomy with a mean followup of 6 years [17]. It was the authors' impression that the previous periacetabular osteotomy did not alter the surgical outcome of the THA based on an improvement of the mean Merle D'Aubigné and Postel score from 11 to 17. Their study, however, lacked a comparison group of patients with THAs without a previous periacetabular osteotomy. Therefore, we compared the outcomes of a group of patients who underwent THA after periacetabular osteotomy with those of a group of matched patients who underwent THA for DDH. We asked: When compared with a matched cohort of patients with THAs done for DDH without a previous periacetabular osteotomy: (1) does THA after periacetabular osteotomy have a higher complication rate, (2) does THA after periacetabular osteotomy have a higher likelihood of resulting in revision THA, (3) does THA after periacetabular osteotomy have comparable improvements in Harris hip score (HHS), and(4) does THA after periacetabular osteotomy have comparable radiographic results?

\section{Patients and Methods}

Institutional review board approval from the participating centers was obtained before initiation of this study. A retrospective review of all patients undergoing a Bernese periacetabular osteotomy with an anterior capsulotomy, if indicated, and without adjunctive femoral osteotomy between 1994 and 2008 was performed at two centers (Mayo Clinic, Rochester, MN, USA, and Washington University, St Louis, MO, USA). Six hundred forty-five periacetabular osteotomies were performed on 562 patients at these institutions. Thirty hips in 29 patients underwent THA after a previous periacetabular osteotomy (5\%); these patients were considered potentially eligible for our study. It is likely that others have undergone conversion to THA elsewhere, so the proportion of patients undergoing conversion may be higher than 5\%, but records regarding any such patients were not available for review. Twenty-three (77\%) of these 30 hips had a periacetabular osteotomy performed through a modified Smith-Petersen approach. Seven $(23 \%)$ of the 30 hips had an abductor takedown and were excluded from our analysis because their clinical outcomes were compromised owing to postoperative the abductor muscle weakness related to the approach. The THA after periacetabular osteotomy group (Fig. 1) was matched for diagnosis (DDH), age within 1 year, sex, and BMI within 2 points. Fifty-two hips were available for matching. The first available match, without a history of periacetabular osteotomy and a minimum 2 years of followup after THA, was chosen for the control group 

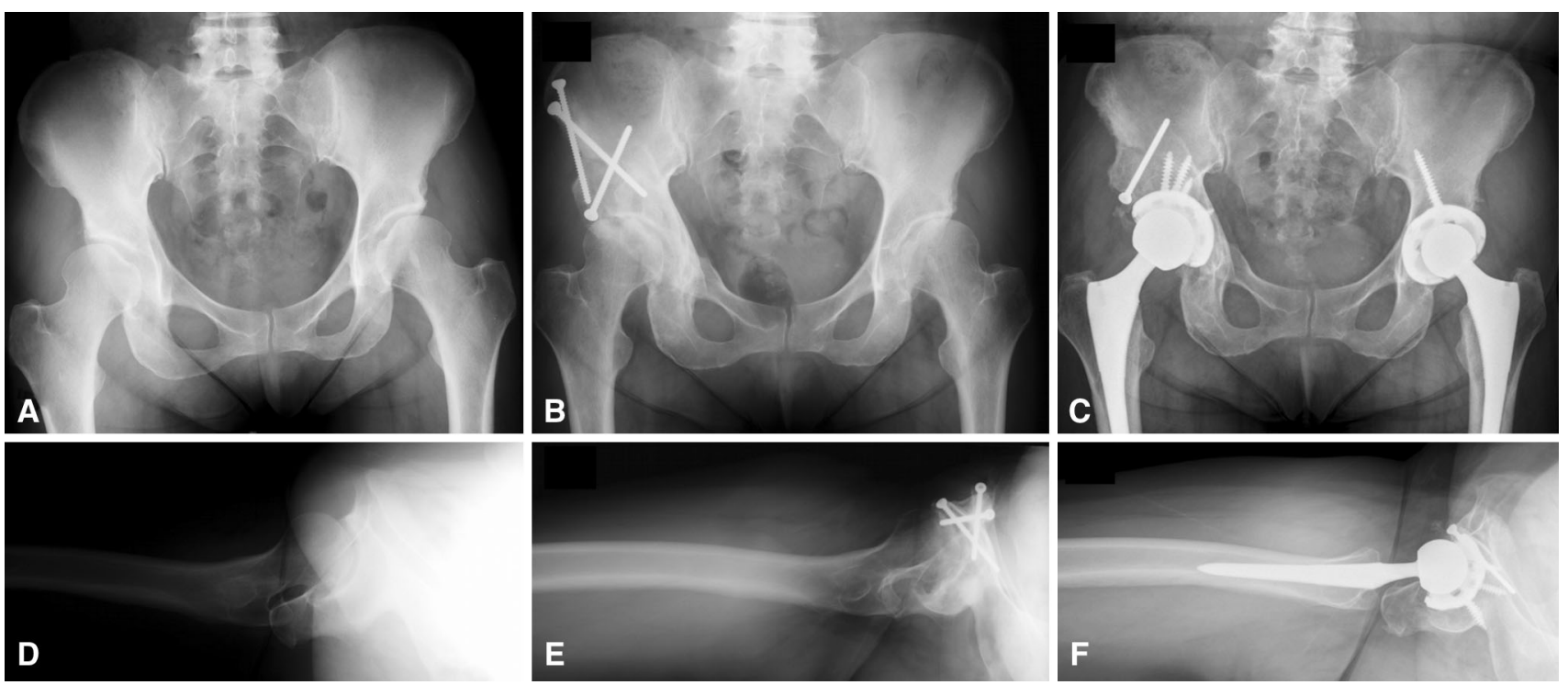

Fig. 1A-F (A) An anterior-to-posterior radiograph of the pelvis shows DDH with a $20^{\circ}$ Tönnis angle and $10^{\circ}$ lateral center-edge angle. (B) An anterior-to-posterior view of the pelvis after periacetabular osteotomy shows the acetabular correction with a $10^{\circ}$ Tönnis angle and $25^{\circ}$ lateral center-edge angle. (C) An anterior-to-posterior radiograph of the pelvis shows conversion to THA after periacetabular osteotomy on the patient's right and without periacetabular

(Table 1). All of the hips undergoing periacetabular osteotomy before the THA were classified as Crowe Grade I, while 22 of the 23 hips (97\%) undergoing THA for DDH were classified as Crowe Grade I and one was Crowe Grade II requiring autograft augmentation. No patients with a history of periacetabular osteotomy required removal of hardware before or during THA. All patients had placement of a press-fit acetabular component and fully coated or proximally coated press-fit femoral implant. All patients in both groups had adjunctive screw fixation of the acetabular component at the time of the THA.

Minimum followup for all patients in both groups was 2 years (mean, $10 \pm 4$ years in the group with THA after periacetabular osteotomy and $6 \pm 4$ years in the group with THA for DDH). The mean time from periacetabular osteotomy to THA was $5 \pm 3$ years, ranging from 1 to 10 years. There was a difference $(p<0.001)$ in final clinical followup between the two groups.

Operative records for all patients who had THAs were reviewed for intraoperative complications, blood loss, and any technical difficulties. THA after periacetabular osteotomy was performed through an anterolateral approach in three hips (17\%) and a posterolateral approach in 20 hips (83\%). THA without periacetabular osteotomy was performed through a lateral approach in four hips (17\%), an anterolateral approach in seven hips (30\%), and a posterolateral approach in 12 hips (52\%). Harris hip scores (HHS) were calculated using the clinical examination osteotomy on the patient's left. (D) A shoot-through lateral view of the hip shows acetabular anteversion. (E) A shoot-through lateral radiograph of the hip after periacetabular osteotomy shows overcorrection with acetabular retroversion. (F) A shoot-through lateral view of the hip shows conversion to THA with resection of the anterior wall and correction of the acetabulum to a more anteverted position.

records and patient-completed questionnaires. Radiographs were reviewed (DFA, LS, PS) before and after periacetabular osteotomy, if necessary, and before and after THA for both groups. The radiographs obtained before and after periacetabular osteotomy were reviewed and the Tönnis angle, anterior center-edge angle, and lateral center-edge angle were reviewed for acetabular correction $[8,21]$. Radiographs obtained before and after THA were reviewed to evaluate for component positioning, loosening, or evidence of impending component failure on the anterior-toposterior pelvis and shoot-though lateral views [4, 5, 24]. Followup for patients having a THA at our institution after the first year is every 5 years, therefore none of the patients who underwent a THA after periacetabular osteotomy is considered lost to followup at the time of this retrospective review. Contact with patients outside their routine surveillance is restricted by our institutional review board for all retrospective studies.

All values are reported as the means \pm standard deviations (SD) where applicable. Comparisons of patientreported outcomes were performed using a t-test. Comparisons of complication and revision rates between groups were performed using a two-sided Fisher's exact test. Analysis of head size variations were performed using a chi-square analysis. A two-tailed post hoc power analysis showed $86 \%$ statistical power to detect an 8-point difference in HHS (our primary outcome measure for distinguishing between the THA after periacetabular 
osteotomy and THA for DDH groups) assuming a SD of 10 points and an $\alpha$ error of 5\%. However, a two-tailed post hoc power analysis showed only $23 \%$ statistical power to detect an $8 \%$ difference in complications with an $\alpha$ error of $5 \%$ indicating that our study was underpowered to make conclusions regarding complications given the small sample size.

\section{Results}

Ten of the 23 hips (44\%) were retroverted after periacetabular osteotomy and at the time of THA. Intraoperatively, six hips with a previous periacetabular osteotomy (26\%) had difficult exposures and dislocations secondary to retroversion of the acetabulum ultimately requiring trimming of the anterior wall in one case or a deficient posterior wall that required bone grafting in another. The mean Tönnis angle before periacetabular osteotomy was $26^{\circ} \pm 8^{\circ}$, the mean anterior center-edge angle was $1^{\circ} \pm 15^{\circ}$, and the mean lateral center-edge angle was $4^{\circ} \pm 9^{\circ}$. The mean Tönnis angle after periacetabular osteotomy was $15^{\circ} \pm 11^{\circ}(\mathrm{p}=0.001)$, the mean anterior center-edge angle was $28^{\circ} \pm 23^{\circ}$ $(\mathrm{p}<0.001)$, and the mean lateral center-edge angle was $20^{\circ} \pm 15^{\circ}(\mathrm{p}<0.001)$. There was no difference in blood loss or operative time ( $\mathrm{p}=0.597$ and 0.155 , respectively) between the groups (Table 1). There was an increase in femoral head size from $28 \mathrm{~mm}$ or less in THA for DDH to $32 \mathrm{~mm}$ or more in THA after periacetabular osteotomy (Table 2).

With the numbers available, there were no differences in the proportion of patients experiencing a major complication between the study groups $(\mathrm{p}=0.489)$. A total of three complications involving operative intervention (13\%) were identified in the THA after periacetabular osteotomy group and none in the THA for DDH group. Two (7\%) patients in the THA after periacetabular osteotomy group sustained dislocations. One patient had multiple recurrent dislocations 1 year after THA attributable to extraarticular bony impingement requiring multiple closed reductions and subsequent revision THA. Another had a dislocation attributable to polyethylene liner wear 11 years after THA and underwent revision THA. Both patients had the primary THA performed through an anterolateral approach. One additional patient with THA after periacetabular osteotomy had a superficial wound-healing problem (3\%) and underwent reoperation.

The proportion of patients undergoing revision THA between the two groups was not different $(p=1.000)$. However, the reasons for revision THA were different between groups. There were four total postoperative revision THAs. Two patients who had THAs after periacetabular osteotomy (7\%) underwent revision THAs
Table 1. Demographics at THA

\begin{tabular}{llll}
\hline Demographic & $\begin{array}{l}\text { THA after periacetabular } \\
\text { osteotomy }(\mathrm{n}=23)\end{array}$ & $\begin{array}{l}\text { THA for DDH } \\
(\mathrm{n}=23)\end{array}$ & $\mathrm{p}$ value \\
\hline $\begin{array}{l}\text { Age at THA } \\
\text { (years) }\end{array}$ & $38 \pm 11$ & $38 \pm 10$ & 0.992 \\
$\begin{array}{l}\text { Sex } \\
1.000\end{array}$ & (male:female) & $6: 17$ & $6: 17$ \\
Height $(\mathrm{cm})$ & $167 \pm 11$ & $162 \pm 17$ & 0.218 \\
$\begin{array}{l}\text { Weight }(\mathrm{kg}) \\
\text { BMI }\left(\mathrm{kg} / \mathrm{m}^{2}\right)\end{array}$ & $29 \pm 6$ & $73 \pm 21$ & 0.202 \\
$\begin{array}{l}\text { Operative time } \\
\text { (minutes) }\end{array}$ & $151 \pm 65$ & $28 \pm 7$ & 0.591 \\
$\begin{array}{c}\text { Blood loss } \\
(\mathrm{mL})\end{array}$ & $630 \pm 383$ & $174 \pm 40$ & 0.155 \\
\hline
\end{tabular}

Values are mean $\pm \mathrm{SD}$; DDH $=$ developmental dysplasia of the hip.

Table 2. Femoral head size

\begin{tabular}{lcc}
\hline $\begin{array}{l}\text { Head size } \\
(\mathrm{mm})\end{array}$ & $\begin{array}{l}\text { THA after periacetabular } \\
\text { osteotomy }\end{array}$ & THA for DDH \\
\hline 22 & 1 & 3 \\
28 & 5 & 16 \\
32 & 13 & 3 \\
36 & 3 & 1 \\
40 & 1 & 0 \\
\hline
\end{tabular}

$\mathrm{DDH}=$ developmental dysplasia of the hip; $\mathrm{p}=0.005$; chisquare $=15.01$.

for dislocation as described above. One patient who had THA for DDH (3\%) underwent revision THA for aseptic loosening of the acetabular component. Another patient who had THA for DDH (3\%) underwent revision THA for problems related to a metal-on-metal articulation.

With the numbers available, there were no differences between the mean HHS for the patients who had THA after periacetabular osteotomy and those who had THA for DDH at latest followup ( $93 \pm 7$ and $95 \pm 10$, respectively; $\mathrm{p}=0.265)$. The mean HHS for patients who had THA after periacetabular osteotomy increased by 26 points from $67 \pm 17$ preoperatively to $93 \pm 7$ at final followup $(\mathrm{p}<0.001)$. The mean HHS increased 44 points for patients who had THA for DDH, from $51 \pm 15$ preoperatively to $95 \pm 10$ at final followup ( $<<0.001$ ). There was a 16-point difference in the mean preoperative HHS $(\mathrm{p}=0.004)$.

There was no difference in acetabular component abduction $(p=0.677)$, but there was a difference in acetabular component anteversion $(p=0.002)$ between the two groups. The acetabular component was placed in $17^{\circ}$ more retroversion during THA after periacetabular osteotomy. The mean acetabular component abduction angle on 
the anterior-to-posterior pelvis radiograph was $44.7^{\circ} \pm 6.6^{\circ}$ and mean acetabular component anteversion on the shootthrough lateral radiograph was $26.3^{\circ} \pm 9.8^{\circ}$ for patients with THA after periacetabular osteotomy. The mean acetabular component abduction angle was $45.6^{\circ} \pm 7.2^{\circ}$ and mean acetabular component anteversion was $36.0^{\circ} \pm 8.2^{\circ}$ for patients with THA for DDH.

\section{Discussion}

The results of periacetabular osteotomy in patients with DDH have been good to excellent at mid- to long-term followup [19]. A select group of patients with DDH, however, will require THA after periacetabular osteotomy, usually for progression of arthritis or unremitting symptoms. THA in patients with DDH often is more complex than in patients with osteoarthritis, and one would expect greater complexity after periacetabular osteotomy and potentially more complications and worse clinical outcomes. Parvizi et al. [17] reported that a previous periacetabular osteotomy does not compromise the results of subsequent THA; however, that study lacked a comparison group of patients without previous periacetabular osteotomy. We compared patients undergoing THA for DDH with patients undergoing THA after a previous periacetabular osteotomy. We found no differences in the proportion of patients experiencing a major complication or revision THA ( $\mathrm{p}=0.489$ and 1.000 , respectively), in mean postoperative HHS at latest followup (93 \pm 7 and $95 \pm 10$, respectively; $\mathrm{p}=0.265)$, and in acetabular component abduction $(\mathrm{p}=0.677)$ between the study groups. However, the acetabular component was placed in $17^{\circ}$ more retroversion during THA after periacetabular osteotomy ( $\mathrm{p}=0.002)$.

There are several limitations to our study. Comparisons were made to answer the study questions based on a retrospective review from prospectively maintained registries of clinical and radiographic information at the two participating centers. However, even with patients tallied across two high-volume centers during almost 15 years, our study was underpowered to detect potentially important differences between the THA after periacetabular osteotomy group and the THA for DDH group. Second, retrospectively analyzed data have several potential limitations including the fidelity of the record, loss to followup, and selection bias. We used a systematic approach to patient selection and a high-fidelity electronic medical record to minimize any of these inadvertent biases. Third, THAs were performed by 13 different orthopaedic surgeons, all fellowship-trained in total joint arthroplasty but each with differing approaches to the THA. Fourth, we estimate $5 \%$ of periacetabular osteotomies will require conversion to THA at 5 years. This represents a potential underestimation of the periacetabular osteotomy conversion rate and is subject to patient and clinician reporting. However, because of the perceived difficulties of conversion to THA and because the institutions included in this study are tertiary referral centers, patients who might require a THA most likely would return to the institution where the periacetabular osteotomy was performed. Finally, there was an important difference in followup duration. Patients in this study undergoing THA for DDH had less time to clinical followup than did the patients who had THA after periacetabular osteotomy. One potentially would expect to see differences in hip scores favoring those with less followup, but despite the differences in followup, the hip scores were comparable.

There were no differences in the proportion of patients experiencing a major complication or revision THA between the study groups $(\mathrm{p}=0.489$ and 1.000 , respectively). Two patients underwent revision THA for instability after THA after periacetabular osteotomy. One patient had multiple recurrent dislocations 1 year after THA because of extraarticular bony impingement requiring multiple closed reductions and subsequent revision THA. Another patient had a dislocation owing to polyethylene liner wear 11 years after THA and underwent revision THA. The shorter followup for patients who had THA for DDH may be responsible for this difference. The revision THAs in the patients who had THA for DDH were related to acetabular cup loosening and problems with a metal-onmetal bearing. The lack of failures on the acetabular side in patients with THA after periacetabular osteotomy supports the thought that the periacetabular osteotomy fragment maintains its blood supply and allows porous acetabular components to ingrow.

There was no difference in the mean postoperative HHS at latest followup between the study groups (93 \pm 7 and $95 \pm 10$, respectively; $\mathrm{p}=0.265$ ). A two-tailed post hoc power analysis showed $86 \%$ statistical power to detect a difference in HHS, our primary outcome measure. There was a difference of 16 points in the mean preoperative HHS at the time of THA after periacetabular osteotomy compared with THA for DDH $(p=0.002)$. One possible explanation is that THA was recommended earlier for the patients who had THA after periacetabular osteotomy because of sensitivity to hip pain resulting in the initial periacetabular osteotomy as opposed to patients with DDH without prior pain requiring operative intervention before THA. Another explanation is that patients with less disability may actually achieve higher postoperative HHS, thus benefiting the THA after periacetabular osteotomy group. In our study, 14 hips were rated excellent; eight were rated good, and one was rated fair. This is comparable to 43 of 45 hips ranging from good to excellent reported by Parvizi et al. [17]. 
There was no difference in the mean acetabular component abduction $(\mathrm{p}=0.677)$, but the mean acetabular component anteversion was decreased by $17^{\circ}(\mathrm{p}=0.002)$ in the THA after periacetabular osteotomy group. The surgeon must be meticulous regarding acetabular positioning that may be compromised or complicated during THA after periacetabular osteotomy. This is highlighted by the one patient with THA after periacetabular osteotomy who had multiple recurrent dislocations because of impingement. In our study, 10 of the 23 hips (44\%) were retroverted after periacetabular osteotomy and at the time of THA. We did not review all 645 radiographs for retroversion after periacetabular osteotomy and therefore cannot comment on whether retroversion leads to hip deterioration. Hip impingement resulting from iatrogenic retroversion is associated with persistent pain and worse outcomes after periacetabular osteotomy $[18,20]$. The high number of retroverted hips in the patients who had THA after periacetabular osteotomy underscores the fact that careful attention to fragment position during periacetabular osteotomy is critical to satisfactory long-term results after periacetabular osteotomy. In our series, as opposed to that of the Parvizi et al. [17], acetabular retroversion presented difficulties at the time of surgery. Trimming the anterior wall was performed to place the acetabular component in the proper position and bone graft was used with a deficient posterior wall. Awareness of potential acetabular structural abnormalities present at the time of THA led to acceptable radiographic positioning of the acetabular component comparable to THA for DDH.

With the numbers available, our study was underpowered to detect a difference in the proportion of postoperative complications or revision THAs between the two groups; however, our study was sufficiently powered to detect a difference in HHS and radiographic component position. Our results are consistent with those of Parvizi et al. [17] who retrospectively reviewed 41 patients ( $45 \mathrm{hips}$ ) after THA for failed periacetabular osteotomy at a mean of 6 years from periacetabular osteotomy to THA. There were two revision THAs for aseptic loosening in their series. Both revisions (for acetabular and femoral loosening) occurred at 5 and 9 years after the index THA. They reported good to excellent results according to the Merle D'Aubigné and Postel scores of 39 of 45 hips; six had fair or poor results and the group included patients who had previous surgery (10 patients) and/or a diagnosis other than DDH alone (five with previous neurologic disease, three had slipped capital epiphyses, and one had Legg-Calvé-Perthes disease). In addition, 24 THAs were done through a transtrochanteric approach indicating the complexity of their cases. They reported that more than $1 / 2$ of their patients had acetabular retroversion after periacetabular osteotomy and before THA but posterior undercoverage was not reported as a problem at the time of THA. In our series, as opposed to that of Parvizi et al. [17], acetabular retroversion presented difficulties at the time of surgery. Trimming of the anterior wall was performed to place the acetabular component in the proper position and bone graft was used for a deficient posterior wall. We found no difference in the mean acetabular component abduction $(\mathrm{p}=0.677)$ and a $17^{\circ}$ decrease in mean acetabular component anteversion with THA after periacetabular osteotomy $(p=0.002)$. Matta et al. [13] retrospectively reviewed eight patients after THA after periacetabular osteotomy through an anterior approach with a mean of 8 years from periacetabular osteotomy to THA. The only complication was an intraoperative calcar fracture which healed uneventfully. All patients who had THA after periacetabular osteotomy in the series of Matta et al. [13] had improved functional scores at a mean followup of 2 years. No direct comparison was made to a control group by Parvizi et al. [17] or Matta et al. [13].

With the numbers available in our study, THA in patients after periacetabular osteotomy offers major improvements in function with acceptable complication and revision rates when compared with THA for DDH in patients without prior periacetabular osteotomy. A THA after a periacetabular osteotomy presents several challenges at the time of surgery and requires preparation and awareness to decrease complications and improve patient outcomes. Acetabular retroversion must be addressed intraoperatively, if encountered, and care must be taken to avoid acetabular component retroversion. However, even with patients' experiences tallied across two high-volume centers for almost 15 years, our study was underpowered to detect potentially important differences between the patients who had THA after periacetabular osteotomy and those who had THA for DDH. Our data are suitable as pilot data for future studies and for systematic reviews. Larger studies are needed to understand how the technical challenges of a THA after periacetabular osteotomy affect the postoperative complication and revision rates.

\section{References}

1. Amendola A, Rorabeck CH, Bourne RB, Apyan PM. Total knee arthroplasty following high tibial osteotomy for osteoarthritis. $J$ Arthroplasty. 1989;4 Suppl:S11-17.

2. Boos N, Krushell R, Ganz R, Muller ME. Total hip arthroplasty after previous proximal femoral osteotomy. J Bone Joint Surg Br. 1997;79:247-253.

3. Broughton NS, Newman JH, Baily RA. Unicompartmental replacement and high tibial osteotomy for osteoarthritis of the knee: a comparative study after 5-10 years' follow-up. $J$ Bone Joint Surg Br. 1986;68:447-452.

4. DeLee JG, Charnley J. Radiological demarcation of cemented sockets in total hip replacement. Clin Orthop Relat Res. 1976;121:20-32.

5. Engh CA, Bobyn JD, Glassman AH. Porous-coated hip replacement: the factors governing bone ingrowth, stress shielding, and clinical results. J Bone Joint Surg Br. 1987;69:45-55. 
6. Ferguson GM, Cabanela ME, Ilstrup DM. Total hip arthroplasty after failed intertrochanteric osteotomy. J Bone Joint Surg Br. 1994;76:252-257.

7. Ganz R, Klaue K, Vinh TS, Mast JW. A new periacetabular osteotomy for the treatment of hip dysplasias: technique and preliminary results. Clin Orthop Relat Res. 1988;232:26-36.

8. Ganz R, Parvizi J, Beck M, Leunig M, Notzli H, Siebenrock KA. Femoroacetabular impingement: a cause for osteoarthritis of the hip. Clin Orthop Relat Res. 2003;417:112-120.

9. Gill T, Schemitsch EH, Brick GW, Thornhill TS. Revision total knee arthroplasty after failed unicompartmental knee arthroplasty or high tibial osteotomy. Clin Orthop Relat Res. 1995;321:10-18.

10. Haddad FS, Bentley G. Total knee arthroplasty after high tibial osteotomy: a medium-term review. J Arthroplasty. 2000;15:597-603.

11. Haslam P, Armstrong M, Geutjens G, Wilton TJ. Total knee arthroplasty after failed high tibial osteotomy long-term followup of matched groups. J Arthroplasty. 2007;22:245-250.

12. Iwase $T$, Hasegawa $Y$, Iwasada $S$, Kitamura S, Iwata H. Total hip arthroplasty after failed intertrochanteric valgus osteotomy for advanced osteoarthrosis. Clin Orthop Relat Res. 1999;364: $175-181$.

13. Matta JM, Stover MD, Siebenrock K. Periacetabular osteotomy through the Smith-Petersen approach. Clin Orthop Relat Res. 1999;363:21-32.

14. Mont MA, Alexander N, Krackow KA, Hungerford DS. Total knee arthroplasty after failed high tibial osteotomy. Orthop Clin North Am. 1994;25:515-525.

15. Mont MA, Antonaides S, Krackow KA, Hungerford DS. Total knee arthroplasty after failed high tibial osteotomy: a comparison with a matched group. Clin Orthop Relat Res. 1994;299: $125-130$.

16. Murphy SB, Ganz R, Muller ME. The prognosis in untreated dysplasia of the hip: a study of radiographic factors that predict the outcome. J Bone Joint Surg Am. 1995;77:985-989.

17. Parvizi J, Burmeister H, Ganz R. Previous Bernese periacetabular osteotomy does not compromise the results of total hip arthroplasty. Clin Orthop Relat Res. 2004;423:118-122.

18. Siebenrock KA, Schoeniger R, Ganz R. Anterior femoro-acetabular impingement due to acetabular retroversion: treatment with periacetabular osteotomy. J Bone Joint Surg Am. 2003;85: 278-286.

19. Steppacher SD, Tannast M, Ganz R, Siebenrock KA. Mean 20year followup of Bernese periacetabular osteotomy. Clin Orthop Relat Res. 2008;466:1633-1644.

20. Tannast M, Pfander G, Steppacher SD, Mast JW, Ganz R. Total acetabular retroversion following pelvic osteotomy: presentation, management, and outcome. Hip Int. 2013;23(suppl 9):14-26.

21. Tönnis D. Congenital Dysplasia and Dislocation of the Hip in Children and Adults. Berlin, Germany: Springer; 1987.

22. Waddell JP, Morton J, Schemitsch EH. The role of total hip replacement in intertrochanteric fractures of the femur. Clin Orthop Relat Res. 2004;429:49-53.

23. Weale AE, Newman JH. Unicompartmental arthroplasty and high tibial osteotomy for osteoarthrosis of the knee: a comparative study with a 12- to 17 -year follow-up period. Clin Orthop Relat Res. 1994;302:134-137.

24. Woo RY, Morrey BF. Dislocations after total hip arthroplasty. $J$ Bone Joint Surg Am. 1982;64:1295-1306. 